\title{
Add-on effect of plum-blossom needling in alopecia areata: a qualitative evidence synthesis
}

\author{
Ting Dai ${ }^{1}$, Ningjing Song ${ }^{2}$, Bin $\mathrm{Li}^{3}$ \\ ${ }^{1}$ Department of Dermatology, Children's Hospital of Fudan University, National Center for Children's Health, Shanghai, China; ${ }^{2}$ Department of \\ Dermatology, Tongren Hospital, Shanghai Jiao Tong University School of Medicine, Shanghai, China; ${ }^{3}$ Shanghai Skin Disease Hospital, Tongji \\ Medical University, Shanghai, China \\ Contributions: (I) Conception and design: T Dai, B Li; (II) Administrative support: N Song; (III) Provision of study materials or patients: T Dai, \\ N Song; (IV) Collection and assembly of data: T Dai, N Song; (V) Data analysis and interpretation: T Dai, N Song; (VI) Manuscript writing: All \\ authors; (VII) Final approval of manuscript: All authors. \\ Correspondence to: Ningjing Song, MD, PhD; Department of Dermatology, Tongren Hospital, Shanghai Jiao Tong University School of Medicine, \\ No.1111, Xian Xia Road, Shanghai, China. Email: snj3367@163.com; Bin Li, MD, PhD. Shanghai Skin disease Hospital, No. 196, Wu Yi Road, \\ Shanghai, China. Email: 18930568129@163.com.
}

Background Alopecia areata (AA) is highly unpredictable, and therapeutic efficacy remains variable, which often prompts patients to seek alternative therapies. Plum-blossom needling has been widely used for thousands of years. The purpose of this meta-analysis was to critically evaluate the add-on effect of plumblossom needling in AA and compared it with that of conventional treatment, which would provide guidance for AA therapy.

Methods: We searched PubMed, Embase, Chinese National Knowledge Infrastructure (CNKI), and WanFang database. We included randomized controlled trials that evaluated the add-on effect of plumblossom needling treatment compared with conventional treatment alone group for AA. The risk of bias was assessed using the Cochrane Handbook for Systematic Reviews of Interventions. We also evaluated the relative risk (RR) and confidence interval (CIs) of response rate (any regrowth, major regrowth, and complete regrowth) between the plum-blossom needling add-on and the conventional control groups.

Results: Finally, eleven articles involving 1,192 patients were included in this meta-analysis (632 cases in the plum-blossom needling combination group and 560 in the conventional control group). The quality of these studies was medium or relatively low. The application of plum-blossom needling add-on therapy had consistent beneficial effects in any regrowth (RR 1.220; 95\% CI: 1.108-1.343; $\mathrm{P}<0.01 ; \mathrm{Q}=48.388$, $\mathrm{I}^{2}=79.334 \%$ ), major regrowth (RR 1.403; 95\% CI: 1.180-1.668; $\mathrm{P}<0.01 ; \mathrm{Q}=68.359, \mathrm{I}^{2}=85.371 \%$ ), and complete regrowth (RR 1.331; 95\% CI: 1.104-1.606; $\mathrm{P}<0.01 ; \mathrm{Q}=31.968, \mathrm{I}^{2}=68.718 \%$ ). Subgroup analysis showed that plum-blossom needling add-on effect remained significant when topical treatment was used. Plum-blossom needling add-on therapy was generally well-tolerated, with no significantly increased risk of Adverse Events (RR 1.391; 95\% CI: 0.475-4.073; P<0.01; Q=1.366, I²=0\%).

Conclusions: Our meta-analysis showed that the combinations of plum-blossom needling provided moderate positive add-one effects in AA patients. Further well-designed research is required to evaluate the optimal plum-blossom needling treatment procedure.

Keywords: Add-on effect, plum-blossom needling, alopecia areata (AA)

Submitted Oct 02, 2020. Accepted for publication Feb 03, 2021.

doi: 10.21037/apm-20-1969

View this article at: http://dx.doi.org/10.21037/apm-20-1969

$\wedge$ ORCID: 0000-0002-5488-2251. 


\section{Introduction}

Alopecia areata (AA) is a common chronic condition, a remitting form of alopecia with an estimated lifetime incidence of nearly $2 \%(1,2)$. Its manifestations range from localized, well-demarcated patches of non-scarring hair loss (patch alopecia, PA) to complete hair loss on the whole scalp (alopecia totalis, AT), or hair loss of the entire body (alopecia universalis, AU). AA is considered a tissuespecific autoimmune disease, and many patients experience disease progression without treatment. AA causes significant emotional stress and psychological issues for patients and their families (3). Due to significant differences in its clinical manifestations and the high unpredictability of disease course, AA is a skin disease difficult to manage, especially its severe and recalcitrant subtypes $(4,5)$. Traditional therapy includes topical or intra-lesional corticosteroids and contact immunotherapy, while other treatment are also applied for severe AA, including systemic corticosteroids and phototherapy (6). Most topical treatment options are considered reasonably safe. The response rate to intralesional triamcinolone acetonide ranged from $56.3 \%$ to $83.3 \%(7,8)$. However, the pooled rate of complete regrowth rate after contact immunotherapy in another study was found to be only $32.3 \%$ (9). Therefore, despite the availability of many treatment options, their efficacy remains variable.

Generally, acupuncture refers to "needle insertion". Shallow puncture is a type of acupuncture that has been widely used in the therapy of various diseases for more than a thousand years in China $(10,11)$. In plum-blossom needling, a hammer-like tool with a head composed of five or seven needles is used. The name originates from the shape of the set of needles employed, which resemble the clusters of stamens in the plum flower during blooming. Plum-blossom needling applies pricking of multiple needles into the skin to tap specific skin areas or acupuncture points. Plum-blossom needles quickly pop up from the skin since their handle is flexible $(12,13)$. This therapy has been widely used for treatment of dermatology diseases (14), among which, plum-blossom needling is most commonly and successfully used as a therapy for AA in combination with conventional treatment. Based on Chinese traditional theory, it can remove "Yu" (stasis) and promoting regeneration. Recent studies also showed that microneedling and fractional lasers therapy improved hair loss, which was considered an evolving treatment area $(15,16)$.

References to expected treatment responses of AA to plum-blossom needling would be helpful in the management of this disease. Therefore, the purpose of this meta-analysis was to critically evaluate the add-on effect of plum-blossom needling in AA treatment and provide guidance for AA therapy. We also examined the consistency of the add-on effect in the application of different conventional treatment options. We present this article in accordance with the PRISMA reporting checklist (available at http://dx.doi.org/10.21037/apm-20-1969).

\section{Methods}

\section{Search strategy}

We searched PubMed, Embase, Chinese National Knowledge Infrastructure (CNKI), and Wanfang databases from inception to 15 December 2020. "Plum-blossom needle", "five-star needle", "seven-star needle" and "alopecia areata" were combined as keywords or MeSH terms. The reference lists included in the present study were reviewed for further identification of potentially relevant studies. Two researchers independently reviewed the literature and extracted data for analysis (D.T. and S.N.J). Cases of inconsistent results or disagreement were resolved by discussion until consensus was achieved. In cases of the lack of consensus between the two researchers, a third, senior investigator resolved the disagreement. Data were extracted that included the publication year, author's name, baseline characteristics of the groups, number of patients, treatment, course of treatment, defined criteria for therapeutic hair regrowth rate, and adverse events.

\section{Study selection}

PICOS criteria were used in this study. The following inclusion criteria were applied: (I) randomized controlled study; (II) treatment of AA; (III) the add-on effect of plumblossom needling was evaluated and compared with that in the control group; (IV) human subjects were enrolled; (V) hair regrowth rate was classified using 4-grade criteria; and (VI) the treatment duration was three months or more. The following exclusion criteria were employed: (I) noncontrolled study; (II) non-randomized study; (III) Plumblossom needling therapy was implemented in both the observation and the control group; (IV) Plum-blossom needling therapy and other treatments were directly compared; (V) duplicated articles in different databases that included the first publication or large-sample data; (VI) review and case report. We set the duration to at least three months, because a treatment course period of less than 
three months was not sufficiently long to accurately evaluate the AA treatment response.

\section{Risk of bias assessments}

Two investigators independently assessed the quality of the included studies using the Cochrane Handbook guidelines. The following domains were evaluated: random sequence generation, allocation concealment, blinding of participants and personnel, blinding of outcome assessment, incomplete outcome data, selective reporting, and other biases. RevMan 5.2 software (The Nordic Cochrane Centre, Copenhagen, Denmark) was used for this assessment.

\section{Statistical analysis}

CMA (Version 2) software was employed to analyze the collected data. Forest plot were generated to present the relative risk (RR) and confidence interval (CIs) of the response rate established in the Meta-analysis; $\mathrm{P}<0.05$ was considered to indicate statistically significant differences. Hair regrowth rate was classified by 4-grade criteria (complete regrowth, major regrowth, any regrowth, and no regrowth).

Heterogeneity was evaluated using Cochran Q and $\mathrm{I}^{2}$ test. The fixed effect model was chosen when heterogeneity was not obvious, whereas the random effect model was utilized when heterogeneity was obvious $\left(\mathrm{I}^{2}\right.$ value was $0.25,0.50$ and 0.75 corresponded to low, moderate, and high heterogeneity respectively). Subgroup meta-analyses were conducted according to basic conventional therapy (with or without topical treatment). Publication bias was also evaluated.

\section{Result}

\section{Study selection}

A total number of 816 articles were identified. After reading the title and the abstract, 562 duplicated and irrelevant articles were excluded. After a detailed evaluation of the 254 full texts, 7 reviews, 20 irrelevant, 119 case reports or case series, 22 articles use plum-blossom needle as basic treatment, 75 not comparable/ randomized study, or with insufficiant data and 4 repetitive articles were excluded. Finally, 11 studies were included (17-27) (Figure 1).

\section{Description of included studies}

Finally, eleven articles with a total number of 1,192 patients were included in this meta-analysis (632 cases in the plum-blossom needling combination group and 560 patients in the conventional group). All studies were singlecenter. Conventional therapies included oral treatment, intralesional corticosteroids, topical Chinese Traditional Medicine (CTM), oral treatment plus intra-lesional corticosteroids, and oral treatment plus topical CTM. Table 1 summarizes the characteristics of the included study.

\section{Risk of bias assessments}

The quality of these studies was medium or relatively low. Three trials used random number table, details of randomization were unclear in other articles. None of the trials mentioned blinding of participants or physicians who performed outcome evaluation (Figure 2).

\section{Meta-analysis}

The add-on effects of the combination of conventional treatment and plum-blossom needling treatment as compared to the conventional treatment alone group were analysed in the 11 included studies. RR Heterogeneity test showed high heterogeneity for any regrowth, major regrowth and complete regrowth, and thus the random effect model was used. In terms of any regrowth, pooled analysis results revealed a value of RR of 1.198 between the treatment and conventional group (95\% CI: 1.103-1.302; $\mathrm{P}<0.01 ; \mathrm{Q}=48.388, \mathrm{I}^{2}=79.334 \%$ ) (Figure 3), showing a significant beneficial effect of the combination of conventional treatment and plum-blossom needling. Compared with the control group, both the major regrowth (RR 1.403; 95\% CI: 1.180-1.668; P<0.01; Q=68.359, $\mathrm{I}^{2}=85.371 \%$ ) (Figure 4 ) and the complete regrowth rates of the combined group increased significantly (RR 1.331; 95\% CI: 1.104-1.606; $\mathrm{P}<0.01 ; \mathrm{Q}=31.968, \mathrm{I}^{2}=68.718 \%$ ) (Figure 5).

Subgroup analysis was performed according to whether the conventional group including topical treatment. In the topical treatment patients, plum-blossom needling consistently showed a positive add-on effect according in any regrowth (RR 1.243; 95\% CI: 1.151-1.342; $\mathrm{P}<0.01$ ), major regrowth (RR 1.387; 95\% CI: 1.243-1.548; $\mathrm{P}<0.01$ ) and complete regrowth (RR 1.341; 95\% CI: 1.117-1.610; $\mathrm{P}<0.01)$. However, in the cases without topical treatment, plum-blossom needling did not exert a significant add-on effect in any regrowth (RR 1.075; 95\% CI: 0.974-1.186; $\mathrm{P}>0.05$ ), major regrowth ( RR 1.148; 95\% CI: 0.935-1.409; $\mathrm{P}>0.05)$ and complete regrowth (RR 1.281; 95\% CI: 0.894 


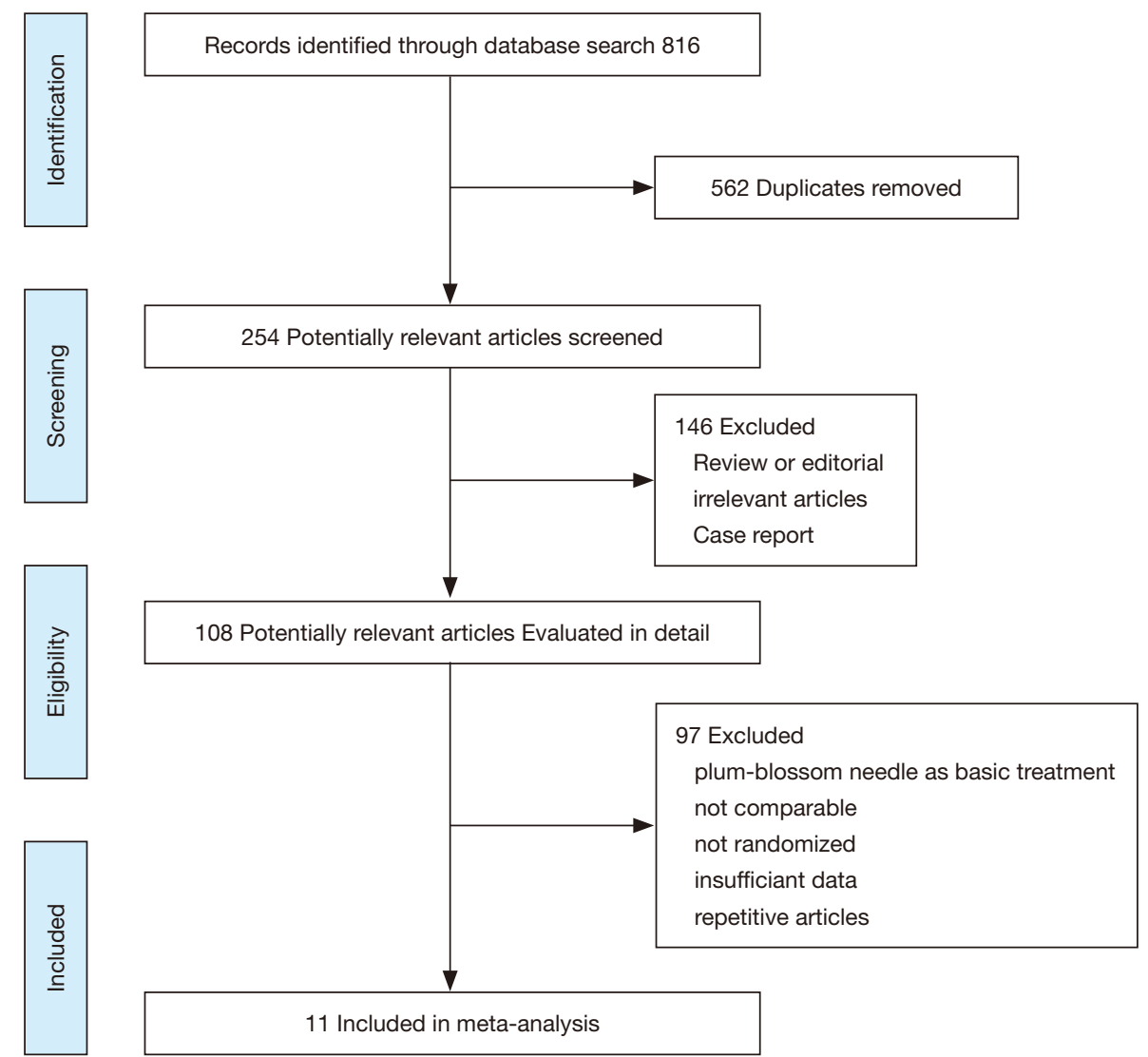

Figure 1 Flow diagram of screened and included studies.

$1.834 ; \mathrm{P}>0.05)$.

Plum-blossom needling add-on therapy was generally well-tolerated. There was no significantly increased risk of AE (RR 1.391; 95\% CI: 0.475-4.073; $\mathrm{P}<0.01 ; \mathrm{Q}=1.366$, $\left.\mathrm{I}^{2}=0 \%\right)$. No serious adverse events were reported in the included studies. Insignificant publication bias existed in the included studies using Egger's test $(\mathrm{P}<0.05)$.

\section{Discussion}

Despite the current availability of many treatment options for AA, the outcome is still unsatisfactory. Thus, patients with AA often seek alternative or unconventional therapies (28). Plum-blossom needling therapy has been widely used for better control of the disease in clinic in combination with conventional treatment options, including topical steroids, topical CTM and systemic therapies.

The results of this meta-analysis revealed that the combination of conventional and plum-blossom needling add-on therapies is more effective than conventional therapy alone. Our results are consistent significant in terms of improved complete regrowth (RR 1.331), major regrowth (RR 1.403), and any regrowth (RR 1.198).

Different acupuncture techniques have been used for thousands of years for the treatment of many dermatological diseases. These techniques include pressneedle acupuncture, warm-needle moxibustion, plumblossom needling, or the application of fire-needles. Longtime clinical practice has shown considerable efficacy of the application of fire-needles in vitiligo treatment, whereas plum-blossom needling is most frequently used in AA cases by repeated tapping of the skin.

In Traditional Chinese Medicine, the mechanism of AA involves mostly dealing with "Yu" (stasis) and "Xu" (deficiency). Several hypotheses had been proposed to explain the mechanism of plum-blossom needling action in hair regrowth. First, it may create minor channels through which topical medications can penetration and reach into the deeper dermis layer, enhancing the effect of the treatment $(12,29)$. Sub-group analysis showed that the add- 


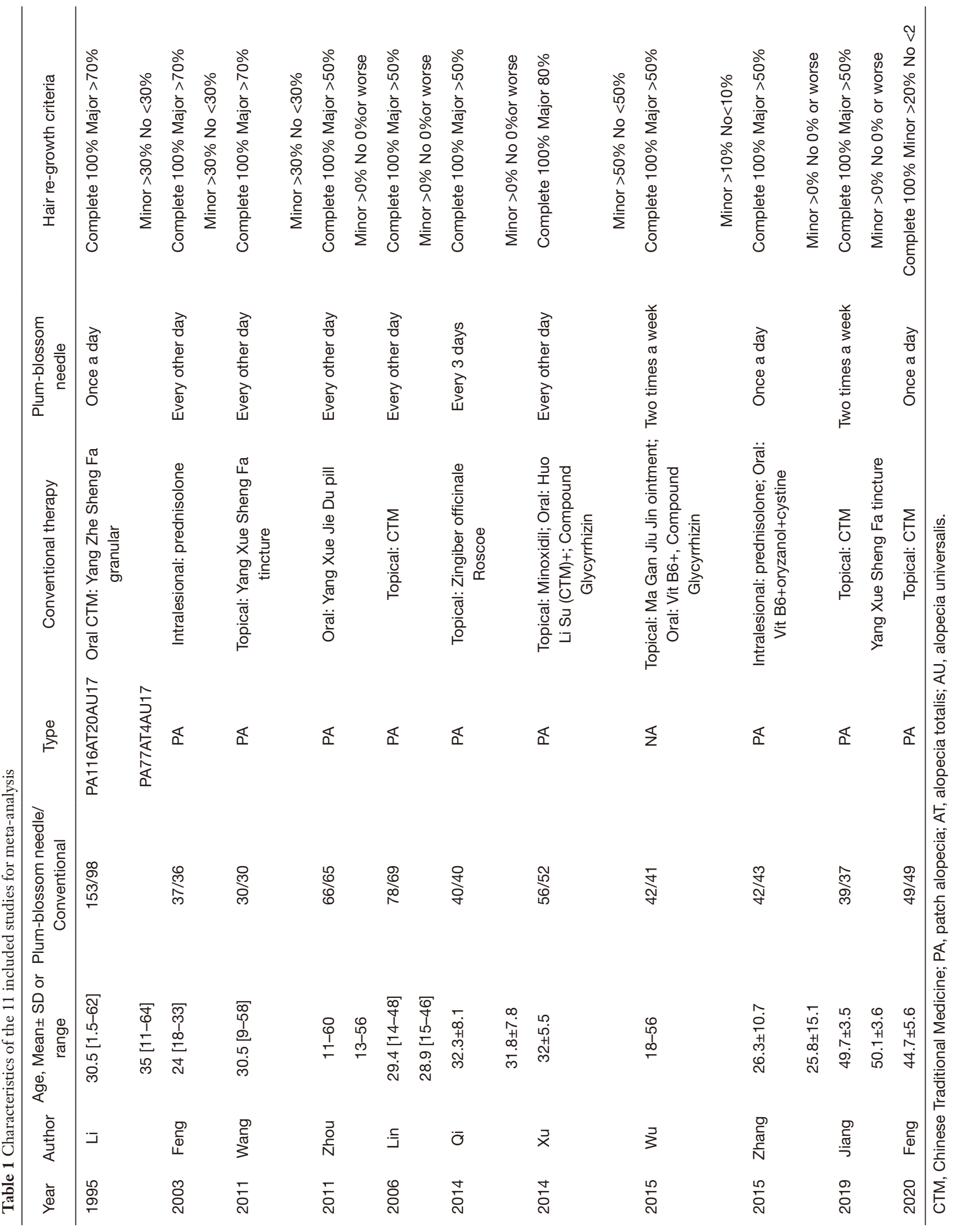




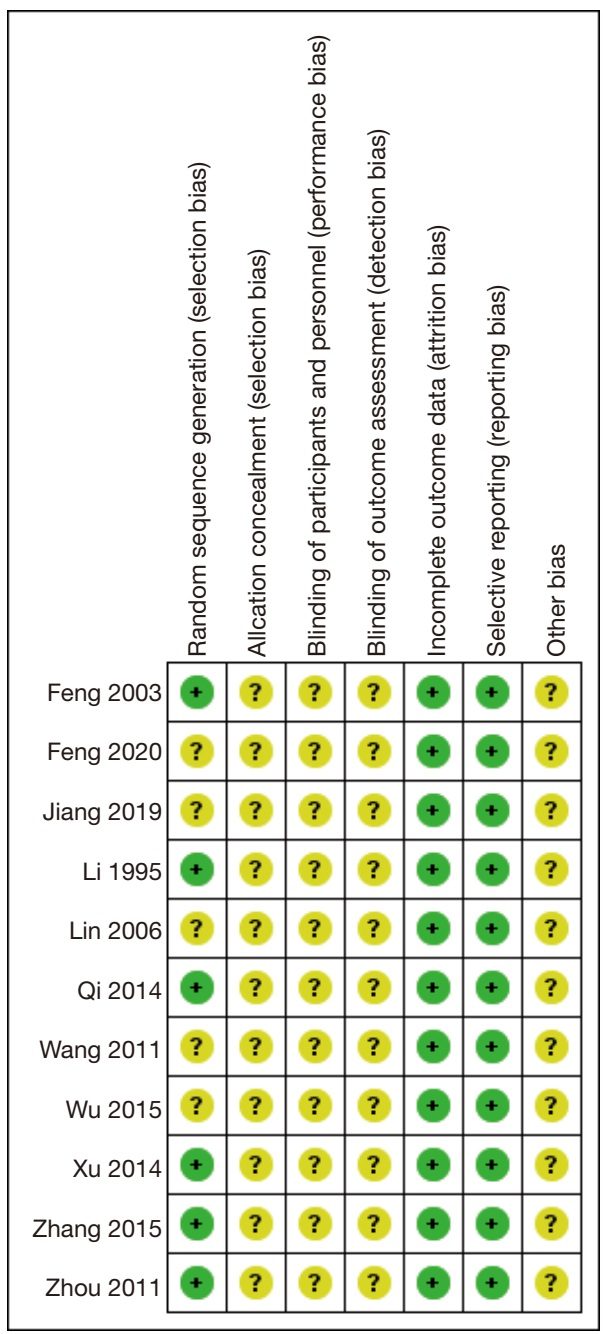

Figure 2 Risk of bias assessments.

on effect of plum-blossom needling remained significant when topical treatment were used. This outcome can be explained by the facilitated drug penetration, which may exert a major role in the mechanism of plum-blossom action. Furthermore, stimulation of hair follicles growth following wound healing has been reported both in animals and humans $(30,31)$. Plum-blossom needling might induce hair growth via activation of the Wnt/-catenin pathway and VEGF, several cytokines and growth factors were secreted in response to the wound-healing response and inflammation (32). Additionally, the repeated tapping of the skin surface could promote skin angiectasis, increasing the regional blood flow around hair follicles and capillaries, thus improving hair regrowth (33). Further investigation is required to elucidate the exact mechanisms of action of plum-blossom combination therapies. In addition to hair regrowth, some patients reported improved sleeping quality and relieved headache. In this respect, Nie reported that plum-blossom needling also regulated the involvement of the neuro-endocrine-immunity network (34). Similarly to plum-blossom needling, microneedling also produced minor rapidly healing punctures and might induce percutaneous collagen and augment transdermal drug delivery. It is first used cosmetically and soon broadened to a wide range of dermatological conditions (35). Fitzpatrick skin types of most Chinese patients belong to III and IV, which means they are characterized by a higher risk of dyspigmentation. Therefore, microneedling and plum-blossom needling is not commonly used in Chinese patients as in those with other skin types for cosmetological reasons.

Plum-blossom needling was generally well-tolerated in the analyzed studies. The most common adverse events were pain and erythema, followed by burning sensation and minor scar. No Serious Adverse Event (SAE) after plum-blossom needling therapy were reported. Plumblossom needling makes a shallow prick, with a minor acupuncture point. Most of the interval skin area were not injured. Therefore, the treated area recovered quickly with minimized adverse events.

The present study has several limitations. First, the patients were not blinded in the included studies since sham treatment simulating plum-blossom needling was not feasible. Second, most publications did not specify the randomization process. Thirdly, there were seldom detailed protocols on the procedure of the therapy applied, such as specifying the distance of vertical tapping and the number of hits needed to produce the desired effect. Fourth, few studies reported long-term follow up result, and a 3-month duration of the follow-up period may not have been sufficient to draw sound conclusions. In addition, the relapse rate was rarely reported in the included investigations. Despite these limitations, our meta-analysis provided the highest-level level of evidence currently available on plumblossom needling add-on effect in AA treatment.

\section{Conclusions}

In conclusion, our meta-analysis showed that the combination of plum-blossom needling and conventional options for AA treatments is safe and more effective than conventional treatments alone. Further well-designed research is required to explore and establish the optimal treatment procedure, duration, and long term efficacy of 
Meta Analysis

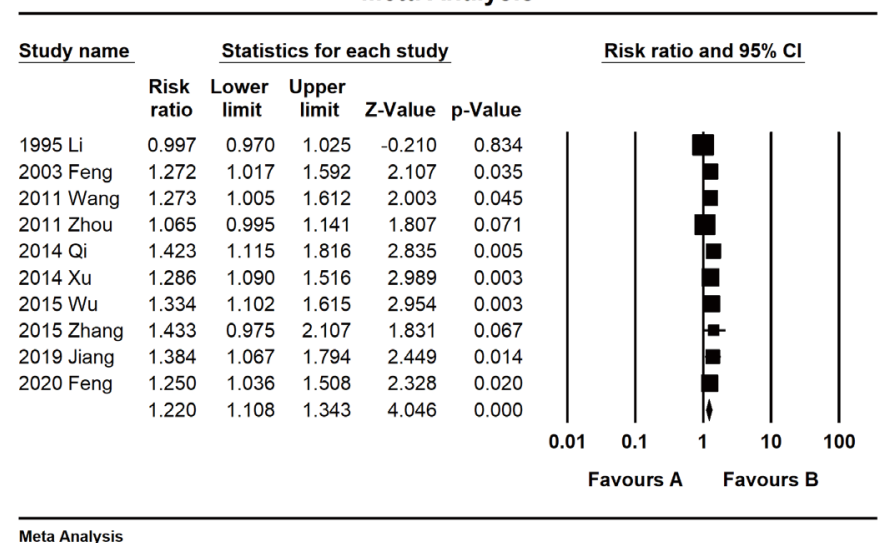

Figure 3 RR of any regrowth between plum-blossom add-on and conventional group. RR, relative risk.

Meta Analysis

\begin{tabular}{|c|c|c|c|c|c|c|c|c|c|}
\hline \multirow[t]{2}{*}{ Study name } & \multicolumn{5}{|c|}{ Statistics for each study } & \multicolumn{4}{|c|}{ Risk ratio and $95 \% \mathrm{Cl}$} \\
\hline & $\begin{array}{l}\text { Risk } \\
\text { ratio }\end{array}$ & $\begin{array}{c}\text { Lower } \\
\text { limit }\end{array}$ & $\begin{array}{c}\text { Upper } \\
\text { limit }\end{array}$ & Z-Value & p-Value & & & & \\
\hline $1995 \mathrm{Li}$ & 0.974 & 0.931 & 1.019 & -1.143 & 0.253 & & & & \\
\hline 2003 Feng & 1.311 & 0.988 & 1.741 & 1.874 & 0.061 & & & & \\
\hline 2006 Lin & 1.272 & 1.067 & 1.516 & 2.685 & 0.007 & & & & \\
\hline 2011 Wang & 1.769 & 1.123 & 2.787 & 2.461 & 0.014 & & & & \\
\hline 2011 Zhou & 1.193 & 1.045 & 1.362 & 2.613 & 0.009 & & & & \\
\hline 2014 Qi & 1.417 & 1.066 & 1.883 & 2.399 & 0.016 & & & & \\
\hline $2014 \mathrm{Xu}$ & 1.424 & 1.095 & 1.852 & 2.635 & 0.008 & & & & \\
\hline $2015 \mathrm{Wu}$ & 1.695 & 1.177 & 2.443 & 2.833 & 0.005 & & & & \\
\hline 2015 Zhang & 1.955 & 1.080 & 3.536 & 2.216 & 0.027 & & & & \\
\hline 2019 Jiang & 1.337 & 0.980 & 1.823 & 1.834 & 0.067 & & & & \\
\hline \multirow[t]{4}{*}{2020 Feng } & 22.500 & 5.776 & 87.655 & 4.487 & 0.000 & & & & \\
\hline & 1.403 & 1.180 & 1.668 & 3.830 & 0.000 & & & $\bullet$ & \\
\hline & & & & & & 0.01 & 0.1 & 10 & 100 \\
\hline & & & & & & \multicolumn{4}{|c|}{ Favours A Favours B } \\
\hline
\end{tabular}

Meta Analysis

Figure 4 RR of major regrowth between plum-blossom add-on and conventional group. RR, relative risk.

Meta Analysis

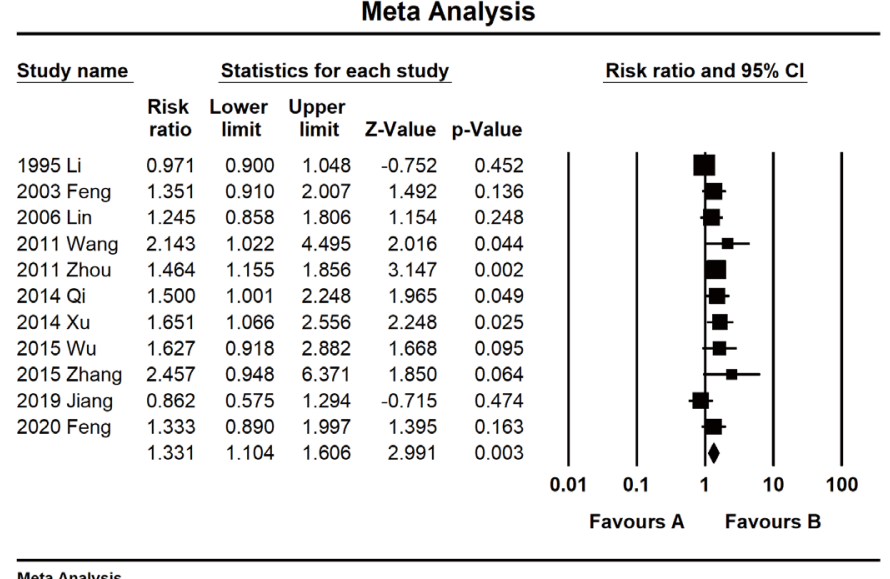

Figure 5 RR of complete regrowth between plum-blossom add-on and conventional group. RR, relative risk. 
plum-blossom needling for AA treatment.

\section{Acknowledgments}

Funding: National Key Research and Development Program of China (no. 2018YFC1705300).

\section{Footnote}

Reporting Checklist: The authors have completed the Narrative Review reporting checklist. Available at http:// dx.doi.org/10.21037/apm-20-1969

Conflicts of Interest: All authors have completed the ICMJE uniform disclosure form (available at http://dx.doi. org/10.21037/apm-20-1969). The authors have no conflicts of interest to declare.

Ethical Statement: The authors are accountable for all aspects of the work in ensuring that questions related to the accuracy or integrity of any part of the work are appropriately investigated and resolved.

Open Access Statement: This is an Open Access article distributed in accordance with the Creative Commons Attribution-NonCommercial-NoDerivs 4.0 International License (CC BY-NC-ND 4.0), which permits the noncommercial replication and distribution of the article with the strict proviso that no changes or edits are made and the original work is properly cited (including links to both the formal publication through the relevant DOI and the license). See: https://creativecommons.org/licenses/by-nc-nd/4.0/.

\section{References}

1. Mirzoyev SA, Schrum AG, Davis MDP, et al. Lifetime incidence risk of alopecia areata estimated at 2.1 percent by Rochester Epidemiology Project, 1990-2009. J Invest Dermatol. 2014;134:1141-2.

2. Tosti A, Bellavista S, Iorizzo M. Alopecia areata: a long term follow-up study of 191 patients. J Am Acad Dermatol 2006;55:438-41.

3. Villasante Fricke AC, Miteva M. Epidemiology and burden of alopecia areata: a systematic review. Clin Cosmet Investig Dermatol 2015;8:397-403.

4. Zaher H, Gawdat HI, Hegazy RA, et al. Bimatoprost versus Mometasone Furoate in the Treatment of
Scalp Alopecia Areata: A Pilot Study. Dermatology 2015;230:308-13.

5. Açıkgöz G, Calışkan E, Tunca M, et al. The effect of oral cyclosporine in the treatment of severe alopecia areata. Cutan Ocul Toxicol 2014;33:247-52.

6. Messenger AG, McKillop J, Farrant P, et al. British Association of Dermatologists' guidelines for the management of alopecia areata 2012. Br J Dermatol 2012;166:916-26.

7. Ustuner P, Balevi A, Özdemir M. Best dilution of the best corticosteroid for intralesional injection in the treatment of localized alopecia areata in adults. J Dermatolog Treat 2017;28:753-61.

8. Amirnia M, Mahmoudi SS, Karkon-Shayan F, et al. Comparative study of intralesional steroid injection and cryotherapy in alopecia areata. Niger Med J. 2015;56:249-52.

9. Lee S, Kim BJ, Lee YB, et al. Hair Regrowth Outcomes of Contact Immunotherapy for Patients With Alopecia Areata A Systematic Review and Meta-analysis. JAMA Dermatol 2018;154:1145-51.

10. Yu J, Ye Y, Li S, et al. The effectiveness and safety of plum-blossom needle therapy for Tourette syndrome: study protocol for a randomized controlled trial. Trials 2015; $16: 320$.

11. Wang X, Han Y, Jin J, et al. Plum-blossom needle assisted photodynamic therapy for the treatment of oral potentially malignant disorder in the elderly. Photodiagnosis Photodyn Ther 2019;25:296-9.

12. Chen J, Zhang Y, Wang P, et al. Plum-blossom needling promoted $\mathrm{PpIX}$ fluorescence intensity from 5 -aminolevulinic acid in porcine skin model and patients with actnic keratosis. Photodiagnosis Photodyn Ther 2016;15:182-90.

13. Wu Y, Wang P, Zhang L, et al. Enhancement of Photodynamic Therapy for Bowen's Disease Using PlumBlossom Needling to Augment Drug Delivery. Dermatol Surg 2018;44:1516-24.

14. Wang P, Zhang L, Zhang G, et al. Successful treatment of giant invasive Cutaneous squamous cell carcinoma by plum-blossom needle assisted photodynamic therapy sequential with imiquimod: case experience. Photodiagnosis Photodyn Ther 2018;21:393-5.

15. Dhurat R, Sukesh M, Avhad G, et al. A randomized evaluator blinded study of effect of microneedling in androgenetic alopecia: a pilot study. Int J Trichology 2013;5:6-11.

16. Majid I, Jeelani S, Imran S. Fractional Carbon Dioxide 
Laser in Combination with Topical Corticosteroid Application in Resistant Alopecia Areata: A Case Series. J Cutan Aesthet Surg 2018;11:217-21.

17. Li XH. Treatment of 251 Alopecia Areata patients with Yang Zhe Sheng Fa granular. Hunan Journal of Chinese Traditional Medicine 1995;11:17-8.

18. Feng H. Plum-blossom needling combined with intralesional prednisolone in the treatment of 37 Alopecia Areata patients. Journal of Dermatology and Venereology $2003 ; 25: 18$.

19. Wang HH, Liu GQ, Zhang HX. Efficacy of Plum-blossom needling and Yang Xue Sheng Fa tincture in Alopecia Areata. Nei Mongol Journal of Traditional Chinese Medicine 2011;12:70.

20. Zhou ZD, Zhou W. Efficacy of Yang Xue Jie Du pills and Plum-blossom needle tapping in Alopecia Areata treatment. Clinical Practice of Integrated Traditional Chinese and Western Medicine 2011;11:61-2.

21. Qi J, Kuang YQ. Efficacy of Plum-blossom needling combined topical ginger usage in Alopecia Areata. Chinese Journal of Aesthetic Medicine 2014;23:2012-3.

22. Xu X, Wang SL. Integrated traditional Chinese, Western Medicine and Plum-blossom needling in Alopecia Areata. Chinese Medical Cosmetology 2014;3:179-80.

23. Wu HY, Tang B. Plum-blossom needling and Ma Gan Jiu Jin ointment in Alopecia Areata treatment. Chinese Journal of Aesthetic Medicine 2015;24:78-9.

24. Zhang GY, Zhang JZ, Liu RQ. Triple therapy of Plumblossom needling, oral and intralesional treatment of Alopecia Areata. Chinese Manipulation and Rehabilitation Medicine 2015;17:27-9.

25. Lin K. The Efficacy of Quincunx-needle Combined with Systemic and Topical Therapy on Treating Alopecia Areata. The Chinese Journal of Dermatovenereology 2006;20:607-8.

Cite this article as: Dai T, Song N, Li B. Add-on effect of plum-blossom needling in alopecia areata: a qualitative evidence synthesis. Ann Palliat Med 2021;10(3):3000-3008. doi: 10.21037/ apm-20-1969
26. Jiang YQ. Effect of Chinese herbal combined with plumblossom needle in adult mild to moderate alopecia areata patients. Journal of Clinical Medical Literature 2019;6:38-9.

27. Feng QD. A study on treating mild alopecia areata in adults with TCM medicine rubbing and plum blossomacupuncture. Clinical Journal of Chinese Medicine 2020;12:130-1.

28. Atanaskova Mesinkovska N. Emerging Unconventional Therapies for Alopecia Areata. J Investig Dermatol Symp Proc 2018;19:S32-3.

29. Mu X, Wang L, Wang L, et al. Plum-blossom needling enhanced the effect of photodynamic therapy on basal cell carcinoma. Photodiagnosis Photodyn Ther 2018;23:339-41.

30. Wier EM, Garza LA. Through the lens of hair follicle neogenesis, a new focus on mechanisms of skin regeneration after wounding. Semin Cell Dev Biol 2020;100:122-9.

31. Rognoni E, Gomez C, Pisco AO, et al. Inhibition of $\beta$-catenin signalling in dermal fibroblasts enhances hair follicle regeneration during wound healing. Development 2016;143:2522-35.

32. Wang L, Gao W, Xiong K, et al. VEGF and BFGF Expression and Histological Characteristics of the BoneTendon Junction during Acute Injury Healing. J Sports Sci Med 2014;13:15-21.

33. Li Q, Xie Y, Zha X. The clinical effect of plum blossom needle acupuncture with qi-invigorating superficiesconsolidating therapy on seborrheic alopecia. Ann Palliat Med 2020;9:1030-6.

34. Nie ZH. Effect of Plum-blossom needling therapy on substance $\mathrm{P}$ level in patients with alopecia areata. Zunyi Medical University, 2012.

35. Cohen BE, Elbuluk N, Microneedling in skin of color: A review of uses and efficacy, J Am Acad Dermatol 2016:74:348-55. 